\title{
Temperatura base para aparecimento de folhas e filocrono da variedade de milho BRS Missões
}

\author{
Base temperature for leaf appearance and phyllochron of the BRS Missões maize variety
}

\author{
Nereu Augusto Streck ${ }^{\mathrm{I}}$ Isabel Lago ${ }^{\text {II }}$ Flávia Kaufmann Samboranha ${ }^{\text {III }}$ Luana Fernandes Gabriel ${ }^{\text {III }}$ \\ Ana Paula Schwantes ${ }^{\text {III }}$ Alfredo Schons ${ }^{\text {IV }}$
}

\begin{abstract}
- NOTA -
RESUMO

O objetivo deste trabalho foi estimar a temperatura base para aparecimento de folhas e o filocrono em uma variedade de milho em várias datas de semeadura e dois anos de cultivo. Um experimento de campo foi realizado em Santa Maria, Rio Grande do Sul (RS), com sete datas de semeadura. Foi utilizada a variedade BRS Missões em dois anos agrícolas consecutivos (2005-06 e 2006-07). O delineamento experimental foi blocos ao acaso com seis repetições. A parcela foi composta por três linhas de $5 \mathrm{~m}$ de comprimento no espaçamento $0,8 \mathrm{~m} \times 0,21 \mathrm{~m}$. Em cada parcela, foram marcadas ao acaso três plantas na linha central nas quais foi realizada semanalmente a contagem do número de folhas

seven sowing dates using the BRS Missões variety in two growing seasons (2005-06 and 2006-07). The experimental design was a completely randomized blocks, with six replications. Plots were three $5 \mathrm{~m}$ rows in a $0.8 \mathrm{~m} \times 0.21 \mathrm{~m}$ spacing. Three plants in the central row of each plot were randomly tagged, and the number of fully expanded leaves (NFE) and the number of leaf tips (NFT) were counted once a week on the tagged plants. The base temperature varied with sowing date from 4.5 to $12^{\circ} \mathrm{C}$ and an average of about $8^{\circ} \mathrm{C}$ was used to calculate thermal time. Phyllochron was higher when calculated on an NFE basis than on a NFT basis, and varied with sowing date from 50.0 to $69.9^{\circ} \mathrm{C}$ day leaf ${ }^{1}$ and from 39.6 to $59.8^{\circ} \mathrm{C}_{\text {day }}$ leaf $^{1}$, respectively, with photoperiod being a likely cause to explain the variation in the phyllochron with sowing date.
\end{abstract} completamente expandidas (NFE) e o total (NFT). A temperatura base variou entre épocas de semeadura de 4,5 a $12^{\circ} \mathrm{C}$, com média próxima de $8^{\circ} \mathrm{C}$, valor que foi usado no cálculo da soma térmica. O filocrono foi maior quando calculado com base no NFE do que com base no NFT e variou com a data de semeadura, de 50,0 a $69,9^{\circ} \mathrm{C}$ dia folha ${ }^{-1}$ e de 39,6 a $59,8^{\circ} \mathrm{C}$ dia folha ${ }^{-1}$, respectivamente, sendo o fotoperíodo uma possível causa da variação do filocrono entre épocas.

Palavras-chave: desenvolvimento, fenologia, soma térmica, graus-dia.

\section{ABSTRACT}

The objective of this study was to estimate the base temperature for leaf appearance and the phyllochron of a maize variety in several sowing dates and two growing seasons. A field experiment was conducted in Santa Maria, RS, with
Key words: development, phenology, thermal time, degree days.

Um parâmetro do desenvolvimento vegetativo das culturas agrícolas é o número de folhas acumuladas na haste principal (NF) (XUE et al., 2004; PAULA et al., 2005; STRECK et al., 2005,2007), que em milho está relacionado com o surgimento de vários estágios de desenvolvimento, a expansão da área foliar e algumas práticas de manejo da cultura (FORSTHOFER et al., 2004; REUNIÃO TÉCNICA ANUAL DE PESQUISA DE MILHO E SORGO DO RS, 2005). Uma das maneiras usadas para calcular o NF

IDepartamento de Fitotecnia, Centro de Ciências Rurais (CCR), Universidade Federal de Santa Maria (UFSM). Avenida Roraima, 1000, 97105-900, Santa Maria, RS, Brasil. E-mail: nstreck1@smail.ufsm.br. Autor para correspondência.

"Programa de Pós-graduação em Engenharia Agrícola, CCR, UFSM, Santa Maria, RS, Brasil.

"IIC Curso de Agronomia, CCR, UFSM, Santa Maria, RS, Brasil.

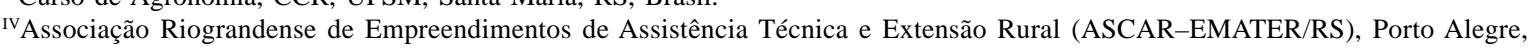
RS, Brasil. 
em modelos matemáticos é pelo conceito do filocrono, que é o intervalo de tempo entre o aparecimento de duas folhas sucessivas em uma haste (WILHELM \& McMASTER, 1995; XUE et al., 2004). A soma térmica geralmente é usada para representar tempo em plantas $\mathrm{e}$, nesse caso, o filocrono tem unidade ${ }^{\circ} \mathrm{C}$ dia folha ${ }^{-1}$ (RUSSELE et al., 1984; STRECK et al., 2005, 2007). Na canônica equação da soma térmica (ST = Tm - Tb), em que Tm é a temperatura média diária do ar e Tb é a temperatura base, abaixo da qual não ocorre desenvolvimento ou o desenvolvimento é tão lento que para fins de cálculo pode ser considerado desprezível (BRUNINI et al., 1976; McMASTER \& WILHELM, 1997; BARBANO et al., 2001), é preciso conhecer o valor da Tb para cada espécie ou genótipo dentro da espécie (GILMORE \& ROGERS, 1958; ARNOLD, 1960).

O uso de variedades de milho é uma alternativa importante na sustentabilidade da atividade agrícola, especialmente nas pequenas propriedades agrícolas familiares. O Rio Grande do Sul (RS) tem regiões com clima Cfa (subtropical) e Cfb (temperado), segundo a classificação de Köppen (MORENO, 1961). $\mathrm{Na}$ maioria das regiões de clima Cfa, a estação de crescimento do milho estende-se de agosto até maio, permitindo até dois cultivos de milho na mesma área por ano agrícola. Assim, é pertinente realizar estudos básicos sobre o desenvolvimento de variedades de milho em diferentes épocas de semeadura. O objetivo detse trabalho foi estimar a temperatura base para aparecimento de folhas e filocrono de uma variedade de milho em várias datas de semeadura e dois anos de cultivo.

Os dados usados neste estudo provêm de um experimento realizado em Santa Maria, RS, (2943'S, $53^{\circ} 42^{\prime} \mathrm{W}$ e altitude: $95 \mathrm{~m}$; clima Cfa) com sete datas de semeadura de milho em dois anos agrícolas consecutivos (2005-06 e 2006-07). A variedade de milho usada foi a BRS Missões, recomendada para os Estados do Rio Grande do Sul, Santa Catarina e Paraná, e indicada para a agricultura familiar (EMBRAPA, 2006). As datas de semeadura foram 21/09/05, 20/10/05, 29/ 11/05, 04/01/06, 07/02/06, 16/03/06 e 12/04/06 no ano agrícola 2005-06 e 23/08/06, 27/09/06, 30/10/06, 30/11/ 06, 07/01/07, 13/02/07 e 15/03/07 no ano agrícola 200607 . Algumas dessas épocas se situam dentro dos períodos recomendados de semeadura do milho na região que são de 11/08 a 20/08 e de 21/11 a 20/01 (REUNIÃO TÉCNICA ANUAL DE PESQUISA DE MILHOE SORGODORS, 2005).

O delineamento experimental foi o de blocos ao acaso com seis repetições. Cada repetição foi composta por uma parcela de três linhas de $5 \mathrm{~m}$ de comprimento no espaçamento de $0,8 \mathrm{~m} \times 0,21 \mathrm{~m}$ e densidade de 6 plantas $\mathrm{m}^{-2}$. O preparo do solo foi $\mathrm{o}$ convencional (duas gradagens). A adubação seguiu a análise de solo, e foi usada irrigação por aspersão para não haver restrição hídrica no solo. Em três plantas por parcela fez-se a contagem semanal do número de folhas completamente expandidas (com o colar visível, NFE) e total (pontas visíveis, NFT) (RITCHIE et al., 1997). A determinação da temperatura base para aparecimento de folhas foi realizada por meio da metodologia do menor quadrado médio do erro (QME) e da regressão linear simples entre o NFE e a soma térmica acumulada (STa), em ${ }^{\circ} \mathrm{C}$ dia, a partir da emergência (SINCLAIR et al., 2004), utilizando-se valores de Tb variando de $0^{\circ} \mathrm{C}$ a $20^{\circ} \mathrm{C}$, com um incremento de $0,5^{\circ} \mathrm{C}$. A STa foi calculada pela equação STa $=\Sigma(\mathrm{Tm}-\mathrm{Tb})(\mathrm{GILMORE} \&$ ROGERS, 1958; ARNOLD, 1960), em que Tmé a temperatura média diária do ar, calculada pela média aritmética entre a temperatura mínima e a temperatura máxima diária, medidas na Estação Climatológica Principal da UFSM, localizada a aproximadamente $250 \mathrm{~m}$ da área experimental. O valor estimado de Tb foi o que teve o menor QME (SINCLAIR et al., 2004). Para a estimativa do filocrono, foi realizada a regressão linear simples entre o NF e a STa a partir da emergência, utilizando-se a Tb estimada anteriormente e o filocrono para cada parcela (considerou-se a média de NFE e NFT das três plantas) foi estimado pelo inverso do coeficiente angular da regressão linear entre NF e STa (XUE et al., 2004; STRECK et al., 2005, 2007). Calculou-se o filocrono com base no NFE e no NFT. Os dados de filocrono foram submetidos à análise da variância e a comparação de médias foi realizada pelo teste Tukey a 5\% de probabilidade de erro. Na análise foi considerado um experimento bifatorial, sendo o fator A as datas de semeadura e o fator D os anos agrícolas (STRECK et al., 2007).

Os valores de $\mathrm{Tb}$ nas datas de semeadura 21/09/05, 20/10/05, 16/03/05 e 12/04/05 (ano agrícola 2005-06) foram $12^{\circ} \mathrm{C}, 10,5^{\circ} \mathrm{C}, 7^{\circ} \mathrm{C}$ e $4,5^{\circ} \mathrm{C}$, respectivamente. Nas outras três datas de semeadura desse ano (29/11/05, 04/01/06 e 07/02/06), a temperatura do ar foi elevada (acima de $15^{\circ} \mathrm{C}$ ) durante o período de emissão de folhas e dessas três épocas não foi possível estimar a Tb nas épocas 29/11/05 e 07/02/06. Quando as temperaturas do ar são elevadas, o método do QME não funciona (SINCLAIR et al., 2004). Na época 04/01/ 06, a Tb estimada foi $8^{\circ} \mathrm{C}$. No ano agrícola 2006-07, foi possível estimar a $\mathrm{Tb}$ apenas para as três primeiras datas de semeadura, sendo os valores estimados de $13^{\circ} \mathrm{C}, 16,5^{\circ} \mathrm{C}$ e $10,5^{\circ} \mathrm{C}$ para as datas de semeadura $23 /$ 08/06, 27/09/06 e 30/10/06, respectivamente, mas esses valores são elevados para milho (YAN \& HUNT, 1999; LOZADA\& ANGELOCCI, 1999), especialmente os dois 
primeiros. Assim, optou-se por usar os valores de $\mathrm{Tb}$ estimados no ano 2005-06 na análise posterior, sendo a média das cinco Tbs estimadas de $8,25^{\circ} \mathrm{C}$. Para fins de cálculo da soma térmica e estimativa do filocrono, considerou-se uma $\mathrm{Tb}=8^{\circ} \mathrm{C}$, valor relatado e usado como Tb em alguns genótipos de milho (YAN \& HUNT, 1999; WHITE, 2001), mas diferente do valor de $10^{\circ} \mathrm{C}$ usado para outros genótipos (LOZADA \& ANGELOCCI, 1999).

A análise de variância resultou em efeito significativo da data de semeadura, do ano agrícola e da interação data*ano para a variável filocrono com base no NFE e com base no NFT. Assim, a análise foi desdobrada dentro de cada fator (Tabela 1). O filocrono com base no NFE foi significativamente maior do que o filocrono com base no NFT em todas as épocas e em todos os anos, sendo, em média, aproximadamente $10^{\circ} \mathrm{C}$ dia folha ${ }^{-1}$ maior para NFE, indicando que o número de folhas em expansão no cartucho aumenta à medida que mais folhas são emitidas na planta.

Houve diferença no filocrono entre as datas de semeadura e a tendência foi similar para ambos os filocronos (NFE e NFT) (Tabela 1). No ano agrícola 2005-06, o maior filocrono $\left(69,9^{\circ} \mathrm{C}\right.$ dia folha ${ }^{-1}$ para NFE e $59,8^{\circ} \mathrm{C}$ dia folha ${ }^{-1}$ para NFT) ocorreu na semeadura mais tardia (12/04/06) e foi maior que nas demais épocas. $\mathrm{O}$ menor valor de filocrono, para NFE, $\left(50,0^{\circ} \mathrm{C}\right.$ dia folha $^{-1}$ ) foi nas semeaduras de 21/09/05 e 04/01/06 e não diferiu do filocrono nas épocas 07/02/06, 16/03/06 e 20/10/05. Já o menor valor de filocrono para NFT $\left(41,6^{\circ} \mathrm{C}\right.$ dia folha $\left.{ }^{-1}\right)$ foi na semeadura de $16 / 03 / 06$ e não diferiu entre as datas 04/01/06, 07/02/06 e 21/09/05. No ano agrícola 2006-07, o maior valor de filocrono com base no NFE $\left(58,8^{\circ} \mathrm{C}\right.$ dia folha $\left.{ }^{-1}\right)$ e no NFT $\left(50,4^{\circ} \mathrm{C}\right.$ dia folha $^{-1}$ ) também ocorreu na última data de semeadura (15/03/07). No entanto, o valor de $58,8^{\circ} \mathrm{C}$ dia folha ${ }^{-1}$ diferiu dos valores de filocrono apenas da semeadura de 30/11/06 para NFE, e o valor de $50,4^{\circ} \mathrm{C}$ dia folha ${ }^{-1}$ não diferiu apenas do filocrono baseado no NFT da semeadura de 13/02/07. Considerando a média das datas de semeadura em cada ano, não houve diferença entre a média de filocrono baseado no NFE nos dois anos, e o filocrono baseado no NFT foi estatisticamente maior em 2005-06 (Tabela 1).

No presente estudo, foi testada a hipótese de que a temperatura do ar pode afetar o filocrono nas diferentes épocas por meioda regressão linear entre filocrono e temperatura média diária do ar durante o período de emissão de folhas (PAULA et al., 2005). Neste estudo, houve uma tendência linear negativa entre as duas variáveis, o que concorda com os resultados de CAO \& TIBBITTS (1995). Porém, o coeficiente de determinação foi baixo, com um $\mathrm{R}^{2}=0,21(P=0,0995)$ para NFE e $\mathrm{R}^{2}=0,25(P=0,0695)$ para NFT e assim esta hipótese foi descartada. Foi testada também a hipótese de o fotoperíodo afetar o filocrono por meio da regressão linear entre filocrono e fotoperíodo médio durante o período de emissão de folhas. As duas variáveis tiveram uma relação negativa, com um $\mathrm{R}^{2}=0,40(P=0,0152)$ para NFE e $R^{2}=0,32(P=0,0344)$ para NFT. A relação negativa entre filocrono e fotoperíodo nessa variedade concorda com a resposta ao fotoperíodo relatada em alguns genótipos de milho (ROOD \& MAJOR, 1980; KINIRY et al., 1983), sendo, assim, o fotoperíodo uma possível causa da variação do filocrono entre as épocas de cultivo nessa variedade de milho.

Tabela 1 - Valores de filocrono $\left({ }^{\circ} \mathrm{C}\right.$ dia folha $\left.{ }^{-1}\right)$ com base no número de folhas expandidas (NFE) e no número total (pontas) de folhas (NTF) do milho, variedade BRS Missões, em sete datas de semeadura, em dois anos agrícolas. Santa Maria, RS, Brasil, $2005-2007$.

\begin{tabular}{|c|c|c|c|c|c|c|}
\hline \multirow{2}{*}{ Data } & \multicolumn{3}{|c|}{ 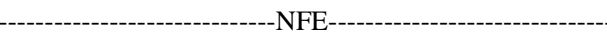 } & \multicolumn{3}{|c|}{-----------------------------NFT--------------------------.. } \\
\hline & 2005-06 & $2006-07$ & Média & 2005-06 & 2006-07 & Média \\
\hline $1^{+}$ & $50,0 \mathrm{Ac}^{*}$ & 51,1 Aab & 50,6 bc & 44,8 Abc & $42,5 \mathrm{Abc}$ & $43,6 \mathrm{~b}$ \\
\hline 2 & $55,3 \mathrm{Abc}$ & $52,0 \mathrm{Aab}$ & 53,6 bc & $47,2 \mathrm{Ab}$ & $44,0 \mathrm{Bbc}$ & $45,6 \mathrm{~b}$ \\
\hline 3 & $57,7 \mathrm{Ab}$ & 52,5 Bab & 55,1 bc & $47,2 \mathrm{Ab}$ & 39,6 Bc & $43,4 \mathrm{~b}$ \\
\hline 4 & 50,0 Ac & $51,0 \mathrm{Ab}$ & 50,4 c & 42,4 Ac & 43,5 Abc & $42,9 \mathrm{~b}$ \\
\hline 5 & 53,5 Abc & 53,5 Aab & 53,5 bc & 43,1 Abc & 42,9 Abc & $43,0 \mathrm{~b}$ \\
\hline 6 & 53,6 Abc & 58,3 Aab & $56,0 \mathrm{~b}$ & $41,6 \mathrm{Bc}$ & 46,5 Aab & $44,1 \mathrm{~b}$ \\
\hline 7 & 69,9 Аа & $58,8 \mathrm{Ba}$ & $64,4 \mathrm{a}$ & 59,8 Аа & $50,4 \mathrm{Ba}$ & 54,9 a \\
\hline Média & $55,7 \mathrm{~A}$ & 53,9 A & & $46,6 \mathrm{~A}$ & 44,2 B & \\
\hline CV (\%) & & 8,00 & & & 5,75 & \\
\hline
\end{tabular}

${ }^{+}$Datas de semeadura: Ano agrícola 2005-06 (1=21/09/05, 2=20/10/05, 3=29/11/05, 4=04/01/06, 5=07/02/06, 6=16/03/06 e 7=12/04/06) e ano agrícola 2006-07 (1=23/08/06, 2=27/09/06, 3=30/10/06, 4=30/11/06, 5=07/01/07, 6=13/02/07 e 7=15/03/07)

*Letras maiúsculas comparam médias na horizontal entre anos agrícolas (2005-06 e 2006-07), e letras minúsculas comparam médias na vertical dentro de cada ano pelo teste Tukey a $5 \%$ de probabilidade de erro. 


\section{REFERÊNCIAS}

ARNOLD, C.Y. Maximum-minimum temperatures as a basis for computing heat units. Proccedings of the Americam Society for Horticultural Sciences, v.76, p.682-692, 1960.

BARBANO, M.T. et al. Temperatura-base e acúmulo térmico no sub-período semeadura-florescimento masculino em cultivares de milho no Estado de São Paulo. Revista Brasileira de Agrometeorologia, v.9, n.2, p.261-268, 2001.

BRUNINI, O. et al. Temperatura base para alface "White Boston”, em um sistema de unidades térmicas. Bragantia, v.35, p.214-219, 1976.

CAO, W.; TIBBITTS, T.W. Leaf emergence on potato stems in relation to thermal time. Agronomy Journal, v.87, p.474477, 1995.

EMBRAPA.Centro Nacional de Pesquisa de Trigo. Milho BRS Missões. Passo Fundo. Capturado em 24 mar. 2006. Online. Disponível em: http://www.cnpt.embrapa.br/culturas/milho/ cultivares/c-missoes.htm.

FORSTHOFER, E.L. et al. Desenvolvimento fenológico e agronônomico de três híbridos de milho em três épocas de semeadura. Ciência Rural, v.34, n.5, p.1341-1348, 2004 .

GILMORE, E.C.; ROGERS, J.S. Heat units as a method of measuring maturity in corn. Agronomy Journal, v.50, n.10, p.611-615, 1958.

KINIRY, J.R. et al. The photoperiod sensitive interval in maize. Agronomy Journal, v.75, p.687-691, 1983.

LOZADA, B.I.; ANGELOCCI, L.R. Determinação da temperatura-base e de graus-dia para a estimativa do subperíodo da semeadura à floração de um híbrido de milho (Zea mays). Revista Brasileira de Agrometeorologia, v.7, n.1, p.3136, 1999.

McMASTER, G.S.; WILHELM, W.W. Growing degree-days: one equation, two interpretations. Agricultural and Forest Meteorology, v.87, n.4, p.291-300, 1997.

MORENO, J.A. Clima do Rio Grande do Sul. Porto Alegre: Secretaria da Agricultura, 1961. 46p.
PAULA, F.L.M. et al. Filocrono da planta de batata cultivar Asterix em diferentes épocas de plantio. Revista Brasileira de Agrometeorogia, v.13, n.2, p.367-374, 2005.

REUNIÃO TÉCNICA ANUAL DE PESQUISA DE MILHO E SORGO DO RS (50 e 33: 2005: Porto Alegre). Indicações técnicas para o cultivo de milho e sorgo no Rio Grande do Sul 2005/2006. Porto Alegre: FEPAGRO/Emater-RS/ ASCAR, 2005. 155p.

RITCHIE, S.W. et al. How a corn plant develops. Ames: Iowa State University of Science and Technology/Cooperative Extention Service, 1997. 21p. (Special Report No.48).

RUSSELE, M.P. et al. Growth analysis based on degree days. Crop Science, v.24, n.1, p.28-32, 1984.

ROOD, S.B.; MAJOR, D.J. Responses of early corn inbreds to photoperiod. Crop Science, v.20, p.679-682, 1980.

SINCLAIR, T.R. et al. Sugarcane leaf area development under field conditions in Florida, USA. Field Crops Research, v.88, p.171-178, 2004.

STRECK, N.A. et al. Estimativa do filocrono em cultivares de trigo de primavera. Revista Brasileira de Agrometeorologia, v.13, n.3, p.423-429, 2005.

STRECK, N.A. et al. Filocrono de genótipos de arroz irrigado em função da época de semeadura. Ciência Rural, v.37, p.323-329, 2007.

WHITE, J.W. Modeling temperature response in wheat and maize. El Batán, México: CIMMYT - International Maize and Wheat Improvement Center, 2001. 60p. (Series 03-01).

WILHELM, W.W.; McMASTER, G.S. Importance of the phyllochron in studying development and growth in grasses. Crop Science, v.35, n.1, p.1-3, 1995.

XUE, Q. et al. Predicting leaf appearance in field-grown winter wheat: evaluating linear and non-linear models. Ecological Modelling, v.175, p.261-270, 2004.

YAN, W.; HUNT, L.A. An equation for modelling the temperature response of plants using only the cardinal temperatures. Annals of Botany, v.84, p.607-614, 1999. 